\title{
13 Lessons for using evidence in policy and practice
}

\author{
Ian Goldman and Mine Pabari
}

\section{Summary}

This final chapter draws together findings and lessons from this study with reference to the analytical framework described in Chapter 3. We reflect on the evidence journeys of the cases in their individual contexts. Diverse sources of evidence were used in across the different cases, and a wide range of evidence use interventions applied. Where an evidence system (such as a national evaluation system) existed, it helped to standardise many of these interventions. Building agreement and trust were key mechanisms leading to change in all the cases, spurring commitment to act. All the case studies resulted in changes in procedures, in some cases extending to changes in policies or budgets. A core message is that evidence use is complex and begins long before an evidence journey starts. Evidence use needs to be planned for and woven into the institutional culture. This needs active facilitation of the process, often in a knowledge brokering role which manages both the supply of and the demand for evidence. Is evidence use the answer to African problems? On its own it is not, but it can make a contribution by helping to lessen the influence of partisan interests and providing some of the answers needed when decisions have to be taken.

\section{Introduction}

This book focuses on improving understanding of how using evidence can help inform and strengthen development policy, programmes and practice in Africa. We looked at the evidence journeys in eight cases, learning from the policy process and how this was accompanied by evidence interventions. The journeys included generation of evidence, activities to promote use and eventual changes (or not) in policy or practice informed by the evidence.

We analyse the processes which support or inhibit evidence use rather than focusing on the sources of evidence, of which much has been written. Four of the cases used evaluations and research synthesis as their key source of evidence and four focused on the role of citizen engagement and evidence from NGOs.

In this chapter we first summarise the findings against the analytical framework (Figure 13.1) ${ }^{1}$ and then reflect on lessons emerging around evidence use. We start by discussing how the contextual influencers and the demand for evidence influenced the way in which the evidence journeys played out across 


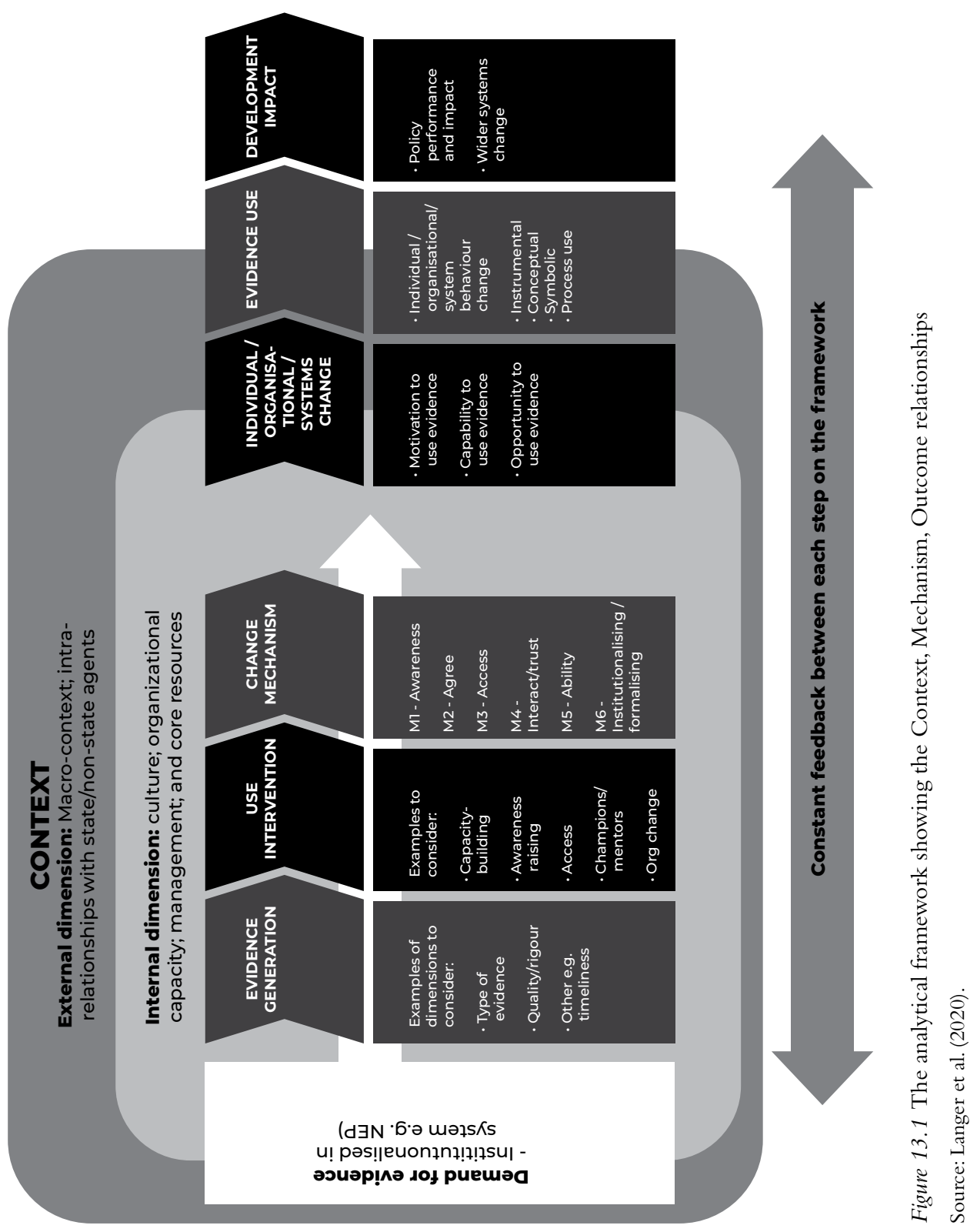


the case studies. We then mention the supply of evidence, evidence use interventions applied in the cases, the change mechanisms these triggered and how these led to immediate outcomes in terms of changes in capabilities, motivation and opportunities and to wider outcomes in terms of changes in policy and practice. Finally we draw out the key messages emerging for promoting evidence-informed policy and practice (EIPP).

\section{Emerging findings}

\section{Contextual factors influencing use}

As succinctly expressed by Weyrauch et al. (2016), 'context matters'. Across all of the case studies, the context within which the evidence journey took place had a significant bearing on how evidence was used.Table 13.1 summarises the contextual influencers identified from across the eight case studies, relating these to the original framework for context. These are discussed in more detail following the table.

Table 13.1 Contextual influencers of evidence use emerging in the case studies

\begin{tabular}{|c|c|c|c|}
\hline Category & $\begin{array}{l}\text { Dimension of } \\
\text { context }\end{array}$ & $\begin{array}{l}\text { Contextual } \\
\text { influencers identified } \\
\text { in the cases }\end{array}$ & Examples from case studies \\
\hline \multirow[t]{9}{*}{ External } & \multirow[t]{7}{*}{ Macro-context } & \multirow{3}{*}{$\begin{array}{l}\text { Significance of the } \\
\text { policy challenge/ } \\
\text { question }\end{array}$} & $\begin{array}{l}\text { Commitments made to international or } \\
\text { regional agreements }\end{array}$ \\
\hline & & & High levels of financial investments \\
\hline & & & Legal requirement for legislative review \\
\hline & & \multirow{3}{*}{$\begin{array}{l}\text { Broader political } \\
\text { and socio- } \\
\text { cultural } \\
\text { environment }\end{array}$} & $\begin{array}{l}\text { Timing, for example, proximity to } \\
\text { election period }\end{array}$ \\
\hline & & & $\begin{array}{l}\text { Space for public participation and civil } \\
\text { society engagement }\end{array}$ \\
\hline & & & $\begin{array}{l}\text { Level of interest and engagement of } \\
\text { stakeholders }\end{array}$ \\
\hline & & \multirow[t]{3}{*}{ Catalysts of change } & Crises \\
\hline & \multirow{2}{*}{$\begin{array}{l}\text { Intra and inter } \\
\text { institutional } \\
\text { linkages }\end{array}$} & & Pressure from development partners \\
\hline & & & Pressure from civil society \\
\hline \multirow[t]{6}{*}{ Internal } & \multirow{6}{*}{$\begin{array}{l}\text { Culture } \\
\text { Organisational } \\
\text { capacity } \\
\text { Management \& } \\
\text { processes } \\
\text { Other resources }\end{array}$} & \multirow{6}{*}{$\begin{array}{l}\text { Institutional } \\
\text { environment }\end{array}$} & Systems and processes \\
\hline & & & Evidence champions \\
\hline & & & Leadership \\
\hline & & & Mandates and capacities \\
\hline & & & Culture - learning and accountability \\
\hline & & & Linkages and relationships \\
\hline
\end{tabular}

Perceived significance of the policy challenge/question

The perceived level of significance of a policy challenge is an important consideration in whether an investment in evidence is seen as worthwhile, and if so, the 
types of use interventions that may be necessary to use to raise the profile of the policy issue. In all of the case studies in this book, countries already had high levels of commitment to the policy issue. For example, a number of the countries had signed international or regional agreements and there was pressure to meet their commitments. ${ }^{2}$ The evidence itself can also help to inform judgements on the significance, such as the magnitude, distribution of effects and causality.

\section{Political and socio-cultural environment}

The broader political and socio-cultural environment influenced whether or not it was worth investing in sourcing and using evidence to support a change process. ${ }^{3}$ For example, how power is distributed and decisions are made had a bearing on evidence use. In some of the cases, presidential proclamations were important in driving policy changes. In other cases, the macro-context enabled public participation and citizen engagement, which allows the decision-making space to be more inclusive. For example, in the agriculture case in Benin, the opening up of spaces to include non-state actors changed the power relationships and resulted in a significant shift in the extent to which evidence was used in the sector to inform decision making and planning.

Perhaps of equal importance was the level of stakeholder engagement and interest as well as the nature of relationships between stakeholders. Where stakeholders were highly fragmented and/or had polarised values and positions, this significantly influenced the level of effort and skill required to manage the evidence process (also discussed later in this section). In the Sanitation, Wildlife and Agriculture cases, citizens felt strongly about the issue, were well organised, had strong capabilities as well as relationships and there was an enabling environment for participation. In these cases, civil society organisations (CSOs) and citizens were an important resource and citizen engagement helped to ensure evidence use.

\section{Catalysers and influencers}

In some cases particular events or actors triggered the need for change, thereby creating an environment conducive to ensuring that the evidence generated was taken seriously and, in these cases, used. For example, a poaching crisis in Kenya and a crisis of education in South Africa provided the impetus and created a sense of urgency which, in these instances, triggered the demand for evidence. In other cases, it was pressure from development partners to generate evidence that was the main trigger for lobbying and advocacy by civil society. We must also recognise that crises may lead to rapid decisions being taken without using the best available evidence, but rather based on beliefs and opinions.

\section{Institutional environment}

Across all the cases, the capabilities of the organisations involved in the evidence journey and the extent to which they were fit for purpose was an important influencer of evidence use. Aspects of institutional capability included the following. 
Competent leadership emerged as important for ensuring that the opportunities for evidence use within the wider context were utilised and barriers navigated. Examples of leadership characteristics identified as being significant included broad-based respect and trust across different stakeholder groups; recognised experience and knowledge of the sector; and having well-established networks and alliances and being seen as politically wise. Another factor was the stability of leadership.

Evidence champions were important in all cases, driving both the generation and use of evidence. Evidence champions were not always in senior leadership positions, although it was helpful when they were; in some cases, they were in civil society. An important lesson was the need for champions to remain in place and to be able to sustain their efforts, as the process of changing policy tends to be lengthy. Champions at the centre of government were important in the evaluations, particularly where the policy issue cuts across sectors, as in the violence case. Knowledge brokers also emerged as playing an important role. ${ }^{4}$

Other important capabilities included skills and knowledge, for example, the ability to access and utilise evidence for decision making and action. Chapter 4 showed that in Benin, Uganda and South Africa, 25\%-33\% of managers do not have the skills to understand and use evaluation recommendations. The limited skills and ability of decision makers and other evidence stakeholders to access, absorb, analyse and synthesise information emerged as a barrier in several of the cases.

Appropriate structures and processes were also important. Organisational silos, competition and overlapping mandates were identified as barriers to evidence use, particularly as coordination and positive relationships are important to enable the dialogue, debate and consensus building necessary for effective use of evidence.

Chapter 4 outlines issues in organisational culture around evidence use in Uganda, Benin and South Africa, indicating that around 50\% of managers support evidence use, but report challenges around hierarchy and fear of punishment for perceived failure. This was confirmed in the case studies where those more open to new ideas and encouraging of change were more likely to enable evidence use. Similarly organisations that are more deliberate in enabling learning and accountability were more likely to utilise evidence than those that do not, for example the Department of Social Development in the violence case study. This was linked to organisational incentives, which emerged as an issue either as a burden (as in the Rapid Response case) or as a motivator (the reward system in budgetary allocations for districts in Ghana).

\section{Demand for evidence}

The next element of the framework in Figure 13.1 is demand for evidence. This may come from government or from other stakeholders. The source of the demand had a bearing on the design of the evidence journey. In some cases demand was institutionalised, for example in a country's national evaluation plans. Where donors were the key demanders, the significance of investing in national and local ownership emerged as being critical to evidence use. In the sanitation case study, civil society was the primary driver behind the demand 
for evidence which led to lessons around the need to put time and energy into ensuring government trust, buy-in and ownership. In all these cases we see a demand for evidence from the evidence users rather than a research push.

\section{Supply of evidence}

Evidence generation is also a part of the framework but not a focus of this book. The different cases show examples of generation through evaluations, research, research synthesis and citizen engagement, and we draw lessons on evidence use across these different methods of generating evidence.

\section{The evidence use interventions}

Table 13.2 shows the range of evidence use interventions that we could see across the cases. In three of the countries there was an NES (national evaluation system) which specified certain interventions and these have been distinguished

Table 13.2 The range of evidence use interventions, as part of, or external to, national evaluation systems

Associated with a NES

- Demand from government

- Evaluation Steering Committee, managing collaboratively the evaluation process

- Process facilitation/knowledge brokering by central government unit

- Capacity-building of key stakeholders around evaluation

- Developing theory of change with stakeholders

- Independent evaluators to ensure credibility

- Validation workshop with stakeholders

- Simple evaluation report

- Management response/ improvement plan

- Quality assessment of the evaluation

- Report public on website

- Approval by Cabinet
Elements seen outside the NES

- Demand from outside government, e.g. from donors/other stakeholders

- Use of international standards and conventions as a reference

- Creation of coalition to support, e.g. civil society coalition in Senegal to support action on tobacco taxation

- Process facilitation/knowledge brokering role of internal unit, either in government (eg Procurement), Parliament (Wildlife) or CSO (Sanitation)

- Scoping study/situation analysis

- Frequent briefings of key stakeholders during the process

- Capacity-building of stakeholders e.g. CSOs

- Sharing drafts amongst stakeholders

- Sharing evidence in accessible formats e.g. short evidence briefs

- Presenting and showcasing evaluation findings at different forums

- Ongoing dialogue in the sector

- Variety of dialogue methods including debates and 1:1 meetings

- Templates and processes for stakeholder inputs

- Proactive outreach and engagement with communities

- Use of peer comparison to promote use 
from other interventions which varied by case. This table provides a useful (but not exhaustive) list to consider in promoting evidence use.

The evidence use interventions that we saw could be applied throughout the process (e.g. maintaining stakeholder involvement), prior to evidence generation (e.g ensuring demand), during the generation (e.g. checking quality and credibility of processes) or after the evidence generation (e.g. dissemination processes).

A key finding that emerges is the importance of facilitation of the evidence journey, often in a knowledge brokering role which manages both supply and demand sides (discussed in Chapter 2). In all cases this brokering role was played by some organisation, sometimes internally, such as an internal monitoring and evaluation (M\&E) unit, and sometimes externally, such as the Centre de la Recherche Economique et Social (CRES), the lead think tank in the tobacco case. Facilitation of the process to promote agreement, ownership, commitment and trust was critical in all the cases. Even where an external entity plays this role, it needs an internal counterpart to work with the evidence. This happens before, during and after the evidence generation process.

Examples from the case studies of these roles include the following:

- Deliberately convening forums and platforms to enable dialogue and debate between the different stakeholder groups.

- Ensuring skilled facilitation, allowing all parties to have an equal voice and creating safe and trusted spaces for meaningful dialogue. In the case studies where this took place this included facilitating negotiation and consensus building, and managing conflict and power dynamics.

- Creating spaces for jointly making sense of the evidence and providing the opportunity for difficult conversations around beliefs and value systems.

- Awareness raising through informal and formal interactions, trainings, meetings and so forth. Dialogue and interaction is also essential to build trust, for example by knowledge brokers with their policy clients - something that ideally needs to happen well before evidence is requested.

- Collaboration in planning and managing the process, so co-creation of the evidence journey. Steps that could be seen were stakeholders working together to frame the problem, develop ToRs, finalise and approve of the methodological framework and timeline, and jointly manage contracts.

- Convening of regular meetings and working hand in hand with programme managers to ensure regular interaction and contact with decision makers, as well as validation workshops with stakeholders.

\section{The change mechanisms triggered by the evidence use interventions}

In order for capabilities/opportunities/motivation to use evidence to be activated, there has to be a change mechanism which inspires people and organisations to do things differently. The list of change mechanisms from Chapter 3 is adapted in Table 13.3, based on what we have seen in this research. Drawing on the experiences of the case studies, we suggest a few changes to the original change mechanisms which are added to the table in italics. 
Table 13.3 The change mechanisms

\begin{tabular}{ll}
\hline Mechanism & Example of interventions to promote use arising in the cases \\
\hline $\begin{array}{l}\text { Awareness of the potential of } \\
\text { evidence (M1) }\end{array}$ & Training senior managers in the public service in South \\
& Africa, Benin and Uganda on evidence (Goldman et al., \\
& 2019) \\
& Training and awareness raising on the potential and value \\
of evidence (e.g. Rapid Response Services) \\
Training of citizen groups in Ghana to analyse and utilise \\
data to demand accountability and better sanitation \\
\\
services as well as in governance and accountability \\
literacy more broadly \\
Establishing dialogue processes to build agreement and \\
commitment \\
Agreement/understanding/ \\
commitment \\
partnerships \\
Pr) & Producing accessible short reports and policy briefs \\
Access to evidence & Workshops \\
(M3) & Dialogue processes \\
Interaction and trust & Knowledge brokering \\
(M4) & Workshops/breakfast meetings \\
& Networks and communities of practice \\
& Capacity-building (e.g. learning by doing, workshops and \\
Ability and confidence & formal training courses) \\
(M5) & Coaching/mentoring \\
& Experiential learning \\
& Online learning \\
Institutionalising/formalising & Use of management responses and improvement plans to \\
(M6) & formalise action needed \\
& Embedded support e.g. knowledge brokering \\
& Institutionalisation of NES \\
& Making public the analysis \\
\hline &
\end{tabular}

Across the cases, building agreement and trust amongst the different players in the evidence journey was key and led to the commitment to act. In some cases, it was necessary to strengthen ability. For example, in the tobacco case, it was necessary to strengthen the ability of technical staff to generate and use evidence, and of politicians to understand the evidence and make decisions. Understanding could also be seen as important in many cases, which links to the importance of conceptual, and not just instrumental, use of evidence.

\section{Outcomes of evidence use in the cases}

Immediate outcomes - changes in capabilities, opportunities and motivation to use evidence

Our analytical framework is based on a behaviour change model where a combination of capability, opportunity and enhanced motivation to use evidence at 
both individual and organisation level leads to behaviour change. In our analytical framework, this corresponds to the immediate outcomes of an evidence process, that is changes in behaviour at the individual, organisational and systems levels which manifest as changes in policy or practice.

In most of the cases strengthening the capability to use evidence emerged as a key component of change. Sometimes the capability was to generate and use evidence, but also we see examples of capacity to advocate for the programme or policy, or for funding and even for the evidence itself.

Motivation to use evidence is an antithesis of the compliance mindset that is common in all these countries. In Chapter 4 we found that around 50\% of managers were motivated to learn and improve policy making, while around $50 \%$ were not. In terms of motivation, Michie's definitions suggest the difference between a reflective motivation based on knowledge and understanding, and an instinctive one, triggered by the topic (Michie et al., 2011). We clearly see examples of increase in motivation of the producer association (PNOPPA) to take forward the agricultural policy in Benin, or the impact of the dialogue in strengthening motivation in the violence case.

In some of the cases with a NES, the institutionalisation of the system created opportunities to use evidence. For example, part of the institutionalisation in South Africa was that national evaluations would go to Cabinet, providing an opportunity for Cabinet to endorse the findings, and this stimulated motivation for the custodian department to use the findings. Some of the mechanisms such as 'trust, agreement, commitment' are also important in opening up opportunities.

\section{And in combination}

In most cases it was the combination of increased capability, motivation and opportunity which made the evidence journey significant and sustained. For example, in the wildlife case, the opportunity to provide inputs into drafting the new Wildlife Act was taken up by a skilled civil society sector and thereafter matched by increased capability of the Kenyan Parliament to manage a participatory process, and to supply and use evidence. The motivation is often driven by key champions, but also by the collective energy from stakeholders. In the Wildlife case, if the motivation of key champions or stakeholders had not been sustained, the Act might have passed, but the drive to take forward the key elements of the Act might have been compromised.

\section{Wider outcomes - changes in policy and practice}

The eight cases selected were purposely selected for being in some way influential, as we sought to understand how and why that influence occurred. The wider outcomes from the different processes resulted mainly from instrumental use of evidence and included policy change, changes in procedures and processes, in budgets and other resources as well as changes in capacity.

Four of the cases showed changes in policies or legislation. In all cases there were changes at process or procedure level, such as guidelines, criteria, thresholds 
for procurement and so forth. Direct evidence of changes in budget allocations was rarer, seen in only two to three of the cases, at least partly because resources to fund the recommendations were not available. In none of the cases were there recommendations to close whole programmes or elements, nor were there findings or recommendations that were significantly controversial/in contradiction to policy makers' beliefs and values.

There were also unintended uses, sometimes arising where there has been conceptual or process use. Unintended uses can have significant long-term impact. For example, the 2009 evaluation of agricultural policy in Benin was not used instrumentally - but the improved understanding from the stakeholders who participated led them to use the evidence in later evaluation and policy processes.

Other unintended uses included:

- $\quad$ The evidence being used to inform other work;

- The lessons being used to widen the work, for example in the wildlife case from community participation in one sector to development of guidelines for public participation with Parliament more generally;

- Strengthening the capacity of particular stakeholders;

- Rebuilding trust between government and stakeholders;

- The evaluation being used for teaching;

- Promoting further research in the area.

\section{What have we learned about promoting the use of evidence?}

In a nutshell, our core message is this:

Evidence use is complex. It begins long before an evidence journey and needs to be planned for and woven into the individual and institutional culture. It is a worthwhile investment.

This research explored interventions to promote evidence use - actions not to generate the evidence but to enable and ensure use. These have to be thought through in an intentional way - what change do you wish to bring about, what change mechanisms need to be triggered and so what evidence use interventions will be needed? Some of the key lessons that emerged in this regard are described below.

\section{The analytical framework is valuable for strengthening evidence use}

For evidence to be utilised, it is important to recognise that evidence use is a journey and not a set of activities focused solely around generation of evidence. The journey involves a series of interconnected processes that can be influenced by the wider environment at all stages. Using the analytical framework, we were able to identify and understand the different stages of the journey and 
develop insights into the relationships between them. In doing so, we recognised the potential of the framework to support evidence generators and users to be more purposive in designing an evidence journey towards ensuring use. Key is understanding the change mechanisms you wish to activate (e.g. agreement/ownership), how this will build the capability/motivation of managers or the opportunity to use evidence, and the evidence use interventions you need to undertake to generate this change.

\section{Evidence use takes place in multiple ways}

We learned the importance of recognising the multiple uses of evidence that can take place (instrumental, conceptual, symbolic, process use, etc.) and the value of designing an evidence journey to be cognisant of these different uses. In focusing simply on evidence and instrumental use, valuable opportunities may be lost. In a number of cases, for example, process and conceptual use were key to bringing about transformational changes that ultimately created the space for positive and sustained impact. Later we discuss the importance of knowledge brokering and facilitation, particularly with regards to process and conceptual use.

\section{Context matters - make sure you understand it}

The evidence journey does not take place in a vacuum and there are multiple factors that influence this journey. We earlier quoted Carol Weiss stating that 'evaluation is a rational enterprise that takes place in a political context' (Weiss, 1993, p. 94). The case studies amplified the importance of understanding this wider context, in line with a core message expressed by Weyrauch et al. (2016). As described earlier, there are contexts where the prevailing political situation is unlikely to allow for evidence use and therefore the investment of an evidence journey may simply not be worthwhile. In other cases, understanding the context can ensure that there is a clearer understanding of relevant entry points and opportunities in the policy process, the change mechanisms necessary to ensure evidence use and the interventions that are most likely to be effective in triggering these mechanisms.

\section{Ensure there is demand}

Much of the writing on EIPP has been by researchers seeking to push their research or evaluation. In this book we take a policy-maker perspective, where policy makers or other stakeholders have requested evidence. In the cases studies we saw a number of ways of ensuring demand:

- Through national evaluation systems requiring evaluations to be done;

- Through policy makers requesting research or research synthesis; 
- Through parliaments requiring citizen inputs into development of legislation;

- Through civil society analysing government data, and the analysed data then being used by government.

The experiences in the cases demonstrate the importance of demand for evidence originating from the evidence users, particularly policy makers. This ensured ownership, strengthened the alignment of the evidence to the policy needs and therefore, ultimately, evidence use.

\section{Ensure credibility, quality and legitimacy in the evidence journey - often it is the messenger as well as the message}

The cases provide examples of different ways in which the credibility of the evidence journey was enhanced.The reputation and track record of the actors generating the evidence as well as those delivering it was extremely important. In a number of cases, consultants were contracted to carry out an evaluation as part of ensuring the independence of the evaluators. Peer reviewers or content experts were also used in several cases to comment on the evidence. The violence case showed the importance of legitimacy in terms of the cultural and racial makeup of the research team. Another important lesson was that transparency and effective communication were important in perceptions of legitimacy of process. A key role for internal and external knowledge brokers was ensuring the quality and credibility of the evidence process, as did stakeholder structures such as steering committees.

\section{Apply evidence use interventions to build capability and motivation}

Passive provision of evidence does not work

Langer et al. (2016) reviewed the facilitators of research uptake and came to the conclusion that research use requires active steps to facilitate access to evidence, to enhance skills in understanding evidence, increase motivation to use evidence, and the formalising of these steps in structures and processes. A passive approach alone, such as seminars or policy briefs will be insufficient.

What we see in the case studies supports these findings. We see where the impact of formalising systems has made a significant contribution, for example through an NES or a formalised citizen engagement process. We see how working to improve decision makers' capability, understanding, motivation and commitment are essential ingredients. In no cases did isolated communication functions play a major role.

\section{The process needs active facilitation and knowledge brokering}

The experience from these cases would suggest that knowledge brokering (also described earlier) is important in the overall evidence journey. These 
roles include keeping policy makers and other stakeholders involved and informed in planning and implementation of the evidence generation process, so keeping them committed and motivated. A key part of the facilitation role was building positive and trusting relationships between stakeholders and with the evidence generation teams. While structures such as steering committees were important, it was essential that they were facilitated effectively. Similarly, where the relationship with the researcher/evaluator was good, there was flexibility in delivery of the evidence, improving recommendations and so forth.

Overall, what emerges is that the process of knowledge brokering is complex, sensitive, and requires strong facilitation skills, and linkages between governmental and non-government stakeholders. These roles currently tend to be under-appreciated and the functions of knowledge brokers in government need to be reviewed to ensure they have the skills and mandates to be successful.

Establishing formal structures to manage the process and maintain ownership of stakeholders

In all but one of the case studies, committees were established to enable different types of engagement across the different stakeholders and sectors, which became a formal expression of the coalitions of stakeholders. These were meant to ensure ownership by key stakeholders in the evidence process. The committees included steering committees to provide overall guidance and decision making and scientific or technical committees involving subject matter specialists from key evidence stakeholder groups (often including development partners). Other forums were sector-driven platforms, such as the Violence Prevention Forum facilitating ongoing dialogue on EIDM in violence prevention in South Africa.

These committees/forums were instrumental to the use of the evidence in a number of different ways. They enabled interaction and the building of relationships between the evidence generators and evidence users, strengthened the abilities of the evidence stakeholders to understand and make sense of the evidence, and helped to ensure the quality, relevance and responsiveness of the evidence, so ensuring a greater sense of ownership of the process as well as the evidence produced.

\section{Build capacity of managers, decision makers and stakeholders}

In a number of cases, investments were made in strengthening the abilities of stakeholders to use evidence. This helped them to play effective roles in the evidence journey. For example, in the sanitation case, citizen groups were trained to analyse and utilise data to demand accountability and better services as well as governance and accountability literacy more broadly. 


\section{Package and communicate the evidence simply and effectively}

Evidence was packaged and communicated in a number of ways to ensure it was appropriate, relevant and accessible to decision makers. Examples included:

- Ensuring the evidence was relevant to the policy concerns, the evidence stakeholders and the wider context;

- The evidence going beyond simply describing a problem to providing practical and realistic solutions;

- Evidence and recommendations being as specific as possible - the more generic, the less likely they are to be used;

- The evidence recognising the values of its recipients. In the case of violence, for example, there was a disconnect in the underlying values of researchers and public servants. Recognising this, the researchers focused the findings on systems and processes rather than engaging with beliefs and values, which, in turn, mitigated risks of rejection and enabled use;

- Formats of reports being readable and accessible, for example using a format for evaluation reports including a 1-page policy summary, 5-page executive summary and 25-page main report format, to ensure reports were readable.

There emerged a number of examples of sensitivity to the dynamics and the need for responsive communication throughout the process. Examples could be seen where findings and recommendations were discussed with higher-level decision makers prior to wider engagement, strengthening their ownership of the evidence and their comfort with the recommendations, so that they would be more likely to implement them.

The experiences of a few of the case studies demonstrate that wider dissemination of the evidence can be as important as the evidence itself, both in terms of how it is shared as well as with whom. In some of these cases, significant effort was made to share the evidence widely using multiple communication media and platforms targeting specific audiences. This included the use of repositories/websites, policy briefs, national dialogues, workshops and seminars. This in turn enabled transparency, ownership and uptake for implementation across multiple stakeholders. In a few of the cases, the evidence was given to trusted and respected individuals to present to stakeholders, as it was recognised that the messenger is often as important as the message itself.

\section{Having an evidence system makes some of the elements automatic}

Five of the eight cases ${ }^{5}$ are from Benin, Uganda and South Africa. These three countries had established a NES which formalise the use of evidence. This includes formalised requirements for evaluations, competencies and standards (benchmarks of evaluation quality, guidelines, peer review mechanisms, etc.). 
In addition, in South Africa formal management responses and improvement plans are required whereby different departments and stakeholders respond to findings and outline how recommendations will be taken up and institutionalised. Developing of the improvement plan again involves stakeholders to ensure quality and ownership of the plans going forward.

Established systems and processes better enable evidence use to be anticipated which, in turn, can improve timely responses to demands.

\section{Lessons on the analytical framework}

The analytical framework we used in Chapter 3 was developed by Langer, deriving from his earlier work (Langer et al., 2016), and that of Vanessa Weyrauch (Weyrauch et al., 2016b). The framework proved very useful in structuring the research and analysing the findings. It evolved slightly in the use. It proved very helpful to be explicit about the behaviour change required for evidence to be used, and to understand what leads to that change. The framework should be valuable for policy makers and practitioners seeking to expand the use of evidence in their work. The context matters framework proved complex to use, and we have simplified it somewhat in our analysis of the contextual influencers. We added additional words in the descriptors, such as commitment and understanding. The version at the beginning of this chapter includes these minor changes.

\section{Conclusions}

Is evidence use the answer to African problems? On its own it is not, but it can make a contribution by helping to lessen the influence of partisan interests in decision making and strengthen its empirical grounding. Evidence can link the implications of decisions to their likely impact on society and ensure that decisions relating to the complex and emergent realities we face are supported or challenged by independent analysis and evaluation. By bringing evidence to the table in a systematic way, anticipating the evidence needs of policy makers, and developing and answering evidence agendas for organisations and the country, it can help to provide some of the answers needed when decisions have to be taken.

The cases we draw from in this book are all examples where evidence has contributed to decision making. They demonstrate that it is possible to use evidence to get improved policies and improved practice, though it is not yet possible to conclude that this results in improved longer-term societal and developmental impact.

The main aim of this research was to find out how can we best facilitate the use of evidence to improve policy and practice and facilitate social outcomes in an African context, and second to test out an analytical framework for understanding evidence use. We conclude that the key factors in the successful use of evidence to improve policy making include understanding context, involving 
stakeholders continuously, ensuring demand for evidence and an appropriate supply, using change mechanisms, building capability and motivation, establishing buy-in at higher levels, and exploiting opportunities within the policy process.

To make evidence more influential requires strengthening the role of knowledge brokers internal and external to government, enabling trusted relationships and creating stronger dialogue between government and stakeholders so that wider influences can inform policy and practice. This requires stronger process skills in government, as well as partnerships with external bodies such as think tanks which have the skills to facilitate and sustain processes.

To do this effectively the key roles of process facilitation and knowledge brokering have to be given more weight, in centre of government and internal evidence/M\&E units, and in the skill sets and job descriptions of the people employed there. This is also true for researchers who seek to influence policy and practice.

\section{Postscript}

\section{Where next?}

The book is part of a process to reflect on African experience and to apply this in policy processes and practice across the continent. The book accompanies other materials, notably videos and policy briefs, intended as resources to help these processes. We hope these resources will inform training and the practice of policy makers, practitioners, parliaments and knowledge brokers. We look forward to continuing the journey with these partners. A luta continua!

\section{Limitations of the research}

The research is built on eight case studies with between 8 and 20 interviews per case. In some cases the researcher of the case study had been involved in the case as a participant observer and so brought considerable richness to the analysis. Clearly, these numbers of interviews are limited. The case studies were undertaken by different researchers so there were some differences in interpretation and how the research was conducted, and how the cases were written up, despite a common template. This has been minimised in that the co-editors then took the cases and turned them into chapters, with the content validated by the authors.

There are several cases of evidence from evaluation and citizen engagement, with only one example of the use of research and one which used research synthesis. However, what we sought to unpack was the process by which evidence use happened, and deliberately take a diversity of evidence generation modalities.

There are some limits in how critical each chapter is, as policy makers involved were co-authors. It was a deliberate strategy to involve the policy 
makers to acknowledge their role and to bring in the richness of their direct experience, but also because the intention was not just to write a book, but to use the content to influence processes in the five countries and the region more widely. Hence the book itself is a change intervention meant to promote interaction and trust between researchers and policy makers, build awareness and commitment to take evidence more seriously in policy and practice, and to strengthen the institutionalisation of evidence. We hope in the process to have built the capability of these policy makers and of the researchers to understand the process by which change happens, increased the motivation of the policy makers to use evidence more actively, and in the remaining part of the project (to June 2020) to support them where opportunities occur to apply the learnings.

\section{Notes}

1 The analytical framework is described in Chapter 1 and discussed in detail in Chapter 3.

2 Such as the ECOWAS countries which had ratified the Framework Convention on Tobacco Control (Chapter 12); and Uganda's commitment to the global guidelines as well as the East, Central and Southern Africa food fortification guidelines and regulatory manual (Chapter 8).

3 In the wildlife case, for example, previous experiences had demonstrated that proximity to an election period meant that there were higher risks of influences and interests other than evidence dominating the decision-making spaces.

4 In the education case, for example, the Chief Directorate Strategic Planning, Research and Coordination had a good reputation as an entity that facilitated the use of evaluations within the Department of Basic Education. In the case of Kenya, on the other hand, there had been a loss of trust in the government during previous policy review processes which meant that there was scepticism around the sincerity of government in its invitation to the wider public to participate in the Wildlife Conservation and Management Act review process. In Benin there was a loss of trust as, while new policies had been developed in response to political changes and these were informed by evidence, these policies did not result in concrete changes in the sector.

5 DBE,VAWC, Procurement, Rapid Response, and Benin.

\section{References}

Goldman, I., Deliwe, C.N., Taylor, S., Ishmail, Z., Smith, L., Masangu, T., Adams, C., Wilson, G., Fraser, D., Griessel, A., Waller, C., Dumisa, S., Wyatt, A. and Robertsen, J. 2019. Evaluation2 - Evaluating the national evaluation system in South Africa: What has been achieved in the first 5 years? African Evaluation Journal, 7(1). https://doi.org/10.4102/aej. v7i1.400

Langer, L., Goldman, I. and Pabari, M. 2020. Analytical framework used to guide case study research. In Using evidence for policy and practice - Lessons from Africa. London: Routledge, Taylor \& Francis Group.

Langer, L., Tripney, J. and Gough, D. 2016. The science of using science: Researching the use of research evidence in decision-making. EPPI-Centre, Social Science Research Unit, UCL Institute of Education, University College London EPPI Centre.

Michie, S., van Stralen, M.M. and West, R. 2011. The behaviour change wheel: A new method for characterising and designing behaviour change interventions. Implementation Science, 6(1), 42. https://doi.org/10.1186/1748-5908-6-42 
Weiss, C.H. 1993. Where politics and evaluation research meet. Evaluation Practice, 14(1), 93-106.

Weyrauch, V., Echt, L. and Suliman, S. 2016. Knowledge into policy: Going beyond 'Context matters'. Politics \& Ideas and the International Network for the Availability of Scientific Publications. 